\title{
Experiência do enfermeiro com o uso da tecnologia em cuidados com o paciente com diabetes no pós cirúrgico
}

\author{
Nurse's experience with the use of technology in the care of patients with diabetes in the \\ postoperative period
}
Experiencia de la enfermera con el uso de la tecnología en el cuidado de pacientes con diabetes en el postoperatorio

Greisiele Aparecida Silva Ferreira Domingues ${ }^{1}$, Claudete Moreschi**, Daiana Foggiato de Siqueira², Letícia Martins Machadoㄹ, Silvana Carloto Andres ${ }^{1}$, Bárbara Belmonte Bedin¹.

\section{RESUMO}

Objetivo: Relatar experiência acadêmica de aplicabilidade da sistematização da assistência de enfermagem e uso de tecnologia educativa de cuidado em pessoas com diabetes no pós-cirúrgico. Relato de experiência: Desenvolvido com pessoas com diabetes, no período pós cirúrgico, em um hospital do interior do Rio Grande do Sul. Inicialmente, identificou-se, nos prontuários, os nomes e os leitos das pessoas que estavam internadas no pós-cirúrgico e que tinham o diagnóstico de Diabetes Mellitus. Foi aplicada a Sistematização da Assistência de Enfermagem e, a seguir, uma tecnologia educativa de cuidado, por meio de uma cartilha educativa que contempla orientações de cuidados para pessoas com diabetes no pós-cirúrgico, utilizando linguagem clara e acessível, além de informações escritas e ilustrações. Considerações finais: Essa experiência proporcionou a reflexão acerca do papel do enfermeiro frente à promoção do autocuidado das pessoas com diabetes. A tecnologia educativa de cuidado por meio de cartilha de orientaçõesàs pessoas com diabetes no pós-cirúrgico poderá estimular as pessoas a procurar mais informações em relação ao conhecimento sobre diabetes, manter os hábitos de vida saudáveis, com vistas à promoção do autocuidado em diabetes no período pós-cirúrgico, melhorar a sua qualidade de vida.

Palavras-chave: Diabetes mellitus, Tecnologia, Enfermagem, Educação em saúde.

\begin{abstract}
Objective: To report academic experience in the application of systematization of nursing care and use of educational care technology in people with diabetes without surgical poster. Experience report: Experience report developed with people with diabetes, in the post-surgical period, in a hospital in the interior of Rio Grande do Sul. Initially, the names and beds of people who were hospitalized in the post-surgical period and who were diagnosed with Diabetes Mellitus were identified in the medical records. The Nursing Care Systematization was applied, followed by an educational care technology, through an educational booklet that includes care guidelines for people with diabetes in the postoperative period, using clear and accessible language, in addition to written and illustrations. Final considerations: This experience provided reflection on the role of nurses in promoting self-care for people with diabetes. The educational technology of care through a booklet of guidelines for people with diabetes in the post-surgical period may encourage people to seek more information regarding knowledge about diabetes, maintain healthy lifestyle habits, with a view to promoting self-care in diabetes in the post-surgical period, improve your quality of life.
\end{abstract}

Key words: Diabetes mellitus, Technology, Nursing, Health education.

\footnotetext{
${ }^{1}$ Universidade Regional Integrada do Alto Uruguai e das Missões, URI, Santiago - Rio Grande do Sul.

*E-mail: clau_moreschi@yahoo.com.br

2Universidade Federal de Santa Maria, UFSM, Santa Maria - Rio Grande do Sul.
}

SUBMETIDO EM: 2/2020

ACEITO EM: $\mathbf{3 / 2 0 2 0}$

PUBLICADO EM: 4/2020

REAS/EJCH | Vol.Sup.n.47 | e3118 | DOI: https://doi.org/10.25248/reas.e3118.2020 Página 1 de 7 


\section{RESUMEN}

Objetivo: Informar sobre la experiencia académica en la aplicación de la sistematización de la atención de enfermería y el uso de tecnología de atención educativa en personas con diabetes sin póster quirúrgico. Informe de experiencia: Informe de experiencia desarrollado con personas con diabetes, en el período posquirúrgico, en un hospital en el interior de Rio Grande do Sul. Inicialmente, los nombres y camas de las personas que fueron hospitalizadas en el período posquirúrgico y que fueron diagnosticadas con diabetes mellitus se identificaron en los registros médicos. Se aplicó la Sistematización de Atención de Enfermería, seguida de una tecnología de atención educativa, a través de un folleto educativo que incluye pautas de atención para personas con diabetes en el postoperatorio, usando un lenguaje claro y accesible, además de escrito e ilustraciones. Consideraciones Finales: Esta experiencia sirvió para reflexionar sobre el papel de las enfermeras en la promoción del autocuidado de las personas con diabetes.La tecnología educativa de la atención a través de un folleto de pautas para personas con diabetes en el período posquirúrgico puede alentar a las personas a buscar más información sobre el conocimiento sobre la diabetes, mantener hábitos de estilo de vida saludables, con el fin de promover el autocuidado en la diabetes en el período posquirúrgico, mejora tu calidad de vida.

Palabras clave: Diabetes mellitus, Tecnología, Enfermería, Educación para la salud.

\section{INTRODUÇÃO}

O Diabetes Mellitus (DM) é uma doença crônica não transmissível (DCNT), a qual é caracterizada por um transtorno metabólico, cujas características são a hiperglicemia e os distúrbios metabólicos (BRASIL, 2013). Essa doença ocorre quando o pâncreas não produz insulina suficiente, um hormônio que regula a glicose no sangue, ou quando o corpo não consegue usar a insulina que produz (WHO, 2018).

Em 2017, a Federação Internacional de Diabetes estimou cerca de 425 milhões de casos de diabetes para faixa etária entre 20 e 79 anos. Para 2045, estima-se cerca de 629 milhões de pessoas com diabetes entre 20 a 79 anos e 693 milhões ao expandir a faixa etária para 18 a 99 anos. Em 2015, no Brasil, o número de pessoas com diabetes na faixa etária de 20 a 79 anos era de cerca de 14,3 milhões de casos, com projeção de 23,3 milhões de diabéticos em 2040 (SBD, 2017).

Os fatores de risco para o surgimento da doença, como a obesidade, têm aumentado com o passar do tempo. A Pesquisa de Orçamentos Familiares (POF) revelou que a frequência de excesso de peso aumentou de 16 para 50\% em homens e de 28 para $48 \%$ nas mulheres nos últimos 36 anos(IBGE, 2011). O diabetes representa também uma causa importante de cegueira, insuficiência renal, amputação de membros inferiores e outras consequências de longo prazo que impactam significativamente, de forma negativa, a qualidade de vida dos portadores da doença (WHO, 2018).

Em 2014, as internações decorrentes do diabetes representaram 4,6\% do total de internações de brasileiros adultos (ROSA RDS, et al., 2018). A Sociedade Brasileira de Diabetes evidencia que os indivíduos com diabetes apresentam maiores taxas de hospitalizações em comparação aos que não têm, além do fato de que essas hospitalizações tendem a ser mais longas, para tratar um mesmo problema, quando comparadas às de pacientes sem diabetes (SBD, 2017).

Neste contexto, as redes de atenção à saúde têm papel importante para oferecer suporte e continuidade na restauração e promoção à saúde das pessoas, contribuindo na melhora da qualidade de vida dos usuários com DM (MORESCHI C et al., 2018). As pessoas que têm doenças crônicas são melhor assistidas quando recebem apoio de várias esferas organizacionais (MENDES EV, 2011). Entre elas, pode-se citar a família, os amigos, os cuidadores, a equipe de saúde multidisciplinar, a organização de saúde e os recursos comunitários (igreja, escola, clube, associação de indivíduos com doenças crônicas, rede web etc.).

Diante do exposto, as tecnologias educativas impressas, como manuais, folhetos, folders, livretos, álbum seriado e cartilhas, são alternativas que sensibilizam a população, levam informações e abrem caminho 
para a promoção da saúde, haja vista que disseminam conhecimento. Essas tecnologias têm como vantagem uma leitura posterior, para pacientes e familiares, servindo como guia de orientações e sanando possíveis dúvidas. Levando em consideração que as tecnologias educativas são ferramentas eficazes, é importante que os enfermeiros apropriem-se de tais tecnologias para dar conta dos desafios relacionados a necessidade de atuação profissional em ações educativas (BENEVIDES JL, et al., 2016).

Deste modo, diante da aplicabilidade de uma tecnologia educativa de cuidado em diabetes, acredita-se que, seja possível contribuir com a diminuição do número de (re)internações de tais indivíduos, o que pode refletir em melhorias na sua qualidade de vida e contribuir para a promoção da saúde. Com base no exposto, o presente estudo tem como objetivo relatar experiência acadêmica de aplicabilidade da sistematização da assistência de enfermagem e uso de tecnologia educativa de cuidado em pessoas com diabetes no pós-cirúrgico.

\section{RELATO DE EXPERIÊNCIA}

Inicialmente, identificou-se, pelos prontuários, os nomes e os leitos das pessoas que estavam internadas no pós-cirúrgico e que tinham o diagnóstico de DM. O primeiro contato com a pessoa participante da experiência foi com a identificação da acadêmica de Enfermagem e a explicação sobre o desenvolvimento do estudo. Logo, questionou-se acerca do interesse da pessoa em participar da experiência, a qual foi desenvolvida com sete pessoas com DM que realizaram um procedimento cirúrgico. Posteriormente, foi aplicada a Sistematização da Assistência de Enfermagem (SAE) embasada nas seguintes etapas do Processos de Enfermagem: Histórico de Enfermagem; Diagnóstico de Enfermagem; Prescrição de Enfermagem. E, a seguir, foi aplicada uma tecnologia educativa de cuidado, por meio de uma cartilha educativa de orientações de cuidado para pessoas com diabetes no pós-cirúrgico.

Os participantes da experiência realizaram diferentes procedimentos cirúrgicos: colecistectomia, apendicectomia, laparotomia, cistostomia, herniorrafia inguinal, nefrectomia e postatectomia. Quanto às varáveis sociodemográficas, quatro participantes são do sexo masculino e três do sexo feminino. A faixa etária foi de 42 a 79 anos de idade, sendo três casados e quatro viúvos. Sobre a ocupação, seis dos sete participantes são aposentados e um é vigilante. Em relação à escolaridade, seis têm ensino fundamental incompleto e um é analfabeto.

Quanto às comorbidades, a maioria das pessoas com DM possuem Hipertensão Arterial Sistêmica (HAS) $(86 \%)$, seguido de cardiopatia (43\%) e hipercolesterolemia (29\%). Em relação aos fatores de risco, identificou-se sedentarismo em $86 \%$ dos participantes, seguido por obesidade $(71,4 \%)$, tabagismo $(29 \%)$ e etilismo (14,2\%). Sobre o número de vezes que se alimenta ao dia, 57\% relataram fazer 3 refeições ao dia, $29 \%$ citaram 2 refeições ao dia e somente 14,2\% disseram que realizam 5 refeições ao dia.

Destaca-se que a maioria possuem complicações macrovasculares, como as cardiopatias, com $43 \%$, tendo como fatores agravantes a hipertensão, a hipercolesterolemia, o sedentarismo, a obesidade e o tabagismo.Quanto às medicações de uso contínuo utilizadas pelas pessoas com DM, $71,4 \%$ utilizam Metformina, 29\% utilizam a Gliblenclamida e 14,2\% a Glimepirida.

A partir dos diagnósticos de Enfermagem supracitados, foram elencadas as seguintes prescrições de enfermagem: controle dos sinais vitais; manter cabeceira elevada a $30^{\circ}$; proporcionar um ambiente tranquilo; realizar curativo em feridas operatórias (FO) e drenos e quantificar drenos; atentar para a integridade da pele e a higiene oral e corporal; massagem de conforto; prevenção de úlcera por pressão com mudança de decúbito a cada 2 horas; atentar para as eliminações fisiológicas; atentar para a ingestão hídrica; avaliar o nível de consciência; observar a cianose de extremidades; atentar para sinais flogísticos em locais de inserção de acesso venoso periférico; observar a presença de edemas e anotar as localizações; observar e anotar o estado de consciência; atentar para oxigenoterapia e controle da glicemia capilar.

Conforme a Associação Norte Americana dos Diagnósticos de Enfermagem (NANDA, 2015), foram elencados os seguintes diagnósticos de Enfermagem, citados no (Quadro 1). 
Quadro 1 - Diagnósticos de Enfermagem das pessoas com diabetes que realizaram um procedimento cirúrgico e participaram da experiência acadêmica.

\begin{tabular}{|c|c|}
\hline Domínio & Diagnósticos de enfermagem mais citados \\
\hline \multirow[t]{5}{*}{$\begin{array}{l}\text { Domínio 1: } \\
\text { Promoção da saúde }\end{array}$} & $\begin{array}{l}\text { Estilo de vida sedentário relacionado a conhecimento deficiente sobre os } \\
\text { benefícios da atividade física }\end{array}$ \\
\hline & $\begin{array}{l}\text { Disposição para controle da saúde melhorado } \\
\text { Relata que deseja melhorar o controle de fatores de risco }\end{array}$ \\
\hline & Manutenção eficaz da saúde relacionada a habilidades motoras prejudicadas \\
\hline & Proteção ineficaz definida por imobilidade ou prejuízo neurossensorial \\
\hline & $\begin{array}{l}\text { Risco da síndrome do idoso frágil devido a fatores como: doença crônica, } \\
\text { fraqueza muscular, hospitalização prolongada, idade }>70 \text { anos, imobilidade } \\
\text { no leito e mobilidade prejudicada }\end{array}$ \\
\hline \multirow{4}{*}{$\begin{array}{l}\text { Domínio 2: } \\
\text { Nutrição }\end{array}$} & Disposição para nutrição melhorada \\
\hline & $\begin{array}{l}\text { Obesidade relacionada à ingestão inadequada de carboidratos e ao } \\
\text { sedentarismo }\end{array}$ \\
\hline & Risco de glicemia instável relacionado ao controle insuficiente \\
\hline & Volume de líquidos deficiente devido à falta de áqua \\
\hline $\begin{array}{l}\text { Domínio 3: } \\
\text { Eliminação e troca }\end{array}$ & Risco de motilidade gastrintestinal disfuncional devido ao DM \\
\hline \multirow{5}{*}{$\begin{array}{l}\text { Domínio 4: } \\
\text { Atividade/repouso }\end{array}$} & Padrão de sono prejudicado devido à mudança no padrão normal do sono \\
\hline & Capacidade de transferência prejudicada devido a processos cirúrgicos \\
\hline & Deambulação prejudicada relacionada à cirurgia e à dor aguda \\
\hline & Desejo de aumentar sua independência \\
\hline & $\begin{array}{l}\text { Déficit no autocuidado para banho, higiene íntima e se vestir relacionado a } \\
\text { desconforto e dor devido a procedimentos cirúrgicos }\end{array}$ \\
\hline \multirow[t]{2}{*}{$\begin{array}{l}\text { Domínio 5: } \\
\text { Percepção/cognição }\end{array}$} & $\begin{array}{l}\text { Conhecimento deficiente definido pelos conhecimentos insuficientes } \\
\text { relacionados por conhecimento insuficiente de recursos }\end{array}$ \\
\hline & Sentimento relacionado à cirurgia e ao quadro clinico \\
\hline \multirow{3}{*}{$\begin{array}{l}\text { Domínio 11: } \\
\text { Segurança/proteção }\end{array}$} & Risco de infecção devido a dispositivos \\
\hline & Risco de integridade da pele prejudicada devido à imobilização física \\
\hline & Risco de queda devido à imobilização \\
\hline
\end{tabular}

Fonte: Domingues GASF, et al., 2020.

A aplicação da tecnologia de cuidado foi produzida a partir de uma cartilha educativa intitulada Orientações para pessoas com diabetes no pós-cirúrgico, a qual foi elaborada a partir das necessidades observadas pela acadêmica no campo prático e profissional, sendo constituída por uma capa, contracapa e 15 páginas de informações de cuidados com o diabetes.

A cartilha foi elaborada com palavras de fácil compreensão e imagens ilustrativas, com explicação de alguns pontos pertinentes sobre DM, dicas de mudanças de hábitos, orientações de autocuidado e cuidados com curativos e dispositivos no pós-cirúrgico. Essas dimensões foram abordadas com a finalidade de melhorar a adesão ao autocuidado e promover a saúde da população com DM depois de uma intervenção cirúrgica. Destaca-se que as imagens ilustrativas são provenientes de busca livre do google com critérios de seleção que incluíram figuras de fácil compreensão, didáticas, atrativas e auto explicativas.

Os temas abordados na cartilha foram: conceito de diabetes; definição de insulina e ação no organismo; complicações agudas (hipoglicemia e hiperglicemia), o que contempla os sintomas e o que fazer diante dessas situações; a importância de manter o índice glicêmico o mais próximo possível do normal para uma melhor recuperação no caso de procedimentos cirúrgicos; hábitos saudáveis, como uma alimentação equilibrada, com frutas, legumes e verduras e a diminuição do consumo de açúcar e sal; importância de uma boa hidratação; exercícios físicos; cuidados com os pés, como a sua higiene e orientações quanto ao uso de calçados e ao corte das unhas; cuidados com a ferida operatória e sinais e sintomas de uma ferida operatória infectada. Também foram apresentados, de forma ilustrativa, alguns drenos e os cuidados relacionados a esses dispositivos, bem como o alerta de sinais e sintomas para FO quando esta estiver infeccionada. 
A construção da cartilha passou por várias etapas, como a seleção dos temas abordados, a seleção de imagens, a escolha do layout, a revisão ortográfica por uma profissional e a organização do layout por um profissional da área. Na revisão ortográfica, foram lapidadas algumas frases para que o texto ficasse mais fácil de ser compreendido pelos participantes. A comunicação torna-se efetiva por meio de uma linguagem compreensível e simples, sendo assim, o profissional de saúde deve atuar como um agente facilitador.

\section{DISCUSSÃO}

Evidenciou-se que $14,2 \%$ analfabetos e $85,8 \%$ tinham apenas o ensino fundamental incompleto. Esses achados podem contribuir com o déficit do autocuidado em pessoas com diabetes. O nível de escolaridade está vinculado ao autocuidado, pois, quanto mais baixo o grau de escolaridade, menor o autocuidado. Esses fatores justificam a falta de compreensão dos cuidados necessários, a alimentação errada e a falta de adesão ao tratamento (CORDEIRO AS, et al., 2017).

O DM tem alta taxa de morbimortalidade associada, principalmente, a complicações microvasculares, como retinopatia, neuropatia e nefropatia, e macrovasculares. Entre as doenças macrovasculares, pode-se citar a doença arterial coronariana (DAC), o acidente vascular cerebral isquêmico (AVC) e a doença arterial periférica (DAP) (SBD, 2017).

Quanto às medicações de uso contínuo utilizadas pelas pessoas com DM, a maioria toma Metformina, seguido de Gliblenclamida. A Metformina é um antidiabético oral, da classe das biguanidas, as quais inibem a produção de glicose pelo fígado e aumentam a sensibilidade dos tecidos corporais à insulina (Smeltzer $S$ e Bare BG, 2002). A Gliblenclamida e a Glimepirida são antidiabéticos orais, do grupo das sulfonilureias, que são dotados de uma potente ação hipoglicemiante (CIMED, 2016). Ressalta-se a importância do enfermeiro orientar as pessoas quanto ao uso correto das medicações, enfatizando que, mesmo com a melhora das taxas glicêmicas, é importante manter a continuidade e não suspender o tratamento sem que haja orientação médica.

A SAE é uma atividade privativa do enfermeiro e utiliza método e estratégia de trabalho científico para a identificação das situações de saúde/doença, subsidiando as ações de assistência de enfermagem. Tratase de uma ferramenta metodológica de assistência do enfermeiro, a qual possibilita a obtenção de dados para o desenvolvimento de ações, com vista à promoção, prevenção, recuperação e reabilitação da saúde do indivíduo, da família e da comunidade.A SAE é uma ferramenta aliada para o cuidado, de forma sistematizada, por meio da qual se conhece o paciente e o seu histórico clínico e cirúrgico, bem como o familiar. A partir disso, pode-se elencar diagnósticos de Enfermagem, prescrever e implantar cuidados, avaliar para verificar os resultados e, posteriormente, se necessário, rever cuidados para que se atinja a meta objetivada (COFEN, 2009).

Notou-seque os participantes não sabiam conceituar o diabetes e a insulina. Essa observação foi oportuna para realizar educação em saúde e, por meio da cartilha educativa impressa, explicar às pessoas sobre a definição da patologia e a ação e a função que a insulina tem no organismo. O DM consiste em um distúrbio metabólico caracterizado por hiperglicemia persistente, decorrente de deficiência na produção de insulina, ou na sua ação, ou em ambos os mecanismos, ocasionando complicações em longo prazo (SBD, 2017).

Foram abordados alguns sinais e sintomas com os quais se devem ficar atentos, como hipoglicemia e hiperglicemia, bem como o que deve ser feito nas situações citadas. Foi contemplada a importância de manter os níveis glicêmicos normais e o quanto isso vai resultar em melhorias para a qualidade de vida e a recuperação no pós-cirúrgico. Observou-se que as pessoas e os familiares desconhecem os sintomas e os sinais das complicações citadas. Na ocasião da orientação, essas informações e a forma de agir foram reforçadas, bem como a importância de sempre informar amigos e familiares que tem diabetes, para, no caso de urgência, eles saberem o que fazer.

Estudoque realizou uma avaliação da intervenção educativa em grupo para diabéticos assistidos em um centro de saúde concluiu que os resultados do grupo que recebeu as orientações a respeito dos aspectos 
gerais do DM foram melhores quando comparados ao outro (BARBOSA L, et al., 2016). Nesse estudo, foi possível notar a importância da educação em saúde para esse público. Ainda, foi possível ver como as pessoas têm dificuldades de compreender o que é um carboidrato e, apesar de alguns já terem ouvido falar que não podem misturar alimentos ricos em açucares complexos, continuam realizando esse ato. Estudo destaca algumas dificuldades das pessoas que têm DM em relação à alimentação, tais como o alto valor dos alimentos saudáveis, a pouca força de vontade, a obrigação de ter que evitar alimentos considerados proibidos e a falta de tempo e conhecimento(LINDEMANN IL, et al., 2016).

Quanto à ingesta hídrica e aos exercícios físicos, a maioria dos participantes confessou que não tem uma ingestão adequada e não pratica exercícios físicos. Em um inquérito de prevalência autorreferida realizado na cidade de Montes Claros, em Minas Gerais, são poucos os usuários que fazem o controle da DM por meio de dieta e/ou atividade física, o que confirma os resultados da prática realizada (DIAS OV, et al., 2016). É competência da Enfermagem ensinar aos pacientes sobre o autocuidado com os pés, como a inspeção diária e a manutenção dos pés limpos e secos, especialmente entre os dedos. Salienta-se a importância de explicar ao paciente sobre o uso de calçados fechados que se adaptem bem aos pés, além de orientar a sempre inspecioná-los antes de calçar (NETA DSR, et al., 2015).

Ainda, reflete-se quanto a importância de manter hábitos saudáveis, uma vez que isto pode se evitar complicações, como amputações, nefropatias, doenças cardíacas, retinopatia diabética e neuropatia diabética. Controlar os níveis glicêmicos é imprescindível para o tratamento do DM. Com o controle metabólico, o paciente se mantém assintomático e se previne das complicações agudas e crônicas, promovendo a qualidade de vida e reduzindo a mortalidade (BRASIL, 2013). O DM modifica o processo de cicatrização e agrava e prolonga o tempo de reparo tecidual, concluindo, essa afecção metabólica tem interferência no processo de cicatrização. Com isso, reforça-se a importância do ensino como educação em saúde e autocuidado (ANDRADE MGL, et al., 2013).

A educação em saúde é considerada uma prática fundamental para as intervenções de promoção da saúde no que se refere às doenças crônicas. A educação em saúde é considerada uma ferramenta para qualificar os conhecimentos, as atitudes e as práticas dos indivíduos, contribuindo no fortalecimento de adesão aos tratamentos em doenças crônicas e melhora da qualidade de vida das pessoas (MOTTA MDC, et al., 2014).

A experiência da aplicabilidade da sistematização da assistência de enfermagem e uso de tecnologia educativa de cuidado em pessoas com DM no pós-cirúrgico possibilitou sanar dúvidas dessa população em relação ao conhecimento sobre $\mathrm{DM}$, e também estimular as pessoas a procurar mais informações e a manter hábitos de vida saudáveis, com vistas à promoção do autocuidado em diabetes no período póscirúrgico.

\section{REFERÊNCIAS}

1. ANDRADE MGL, et al. Evidências de alterações do processo de cicatrização de queimaduras em indivíduos diabéticos: revisão bibliográfica. Revista Brasileira de Queimaduras. 2013;12(1):42-8.

2. BARBOSA L, et al. Avaliação da intervenção educativa em grupo para diabéticos assistidos em um Centro de Saúde Escola. Revista Enfermagem UERJ. 2016;24(2):4968.

3. BENEVIDES JL, et al. Construção e validação de tecnologia educativa sobre cuidados com úlcera venosa. Revista da Escola de Enfermagem da USP. 2016;5(2):306-312.

4. BRASIL. Ministério da Saúde (BR). Estratégias para o cuidado da pessoa com doença crônica: Diabetes Mellitus. Brasília: Ministério da Saúde; 2013.

5. CIMED. Glimepirida: Onefarma Indústria Farmacêutica LTDA: comprimidos: 2 e 4 mg 2016. Disponível em: http://www.anvisa.gov.br/datavisa/fila_bula/frmVisualizarBula.asp?pNuTransacao=27586062016\&pldAnexo=41957 51. Acesso em: 23 nov. 2018.

6. CONSELHO FEDERAL DE ENFERMAGEM - COFEN (BR). Resolução COFEN n 564/2017. Aprova o novo Código de Ética dos Profissionais de Enfermagem. Disponível em: http://www.cofen.gov.br/resolucao-cofen-no5642017_59145.html. Acesso em: 23 nov. 2018. 
7. CONSELHO FEDERAL DE ENFERMAGEM - COFEN(BR). Resolução COFEN-272/2002: revogada pela Resolução COFEN no 358/2009. Dispõe sobre a Sistematização da Assistência de Enfermagem - SAE - nas Instituições de Saúde Brasileiras. 2002. Disponível em: http://www.cofen.gov.br/resoluo-cofen-2722002-revogadapela-resoluao-cofen-n 3582009_4309.html. Acesso em: 23 nov. 2018.

8. CORDEIRO AS, et al. Conhecimento e atitude de pacientes com diabetes mellitus da Atenção Primária à Saúde. Escola Anna Nery Revista de Enfermagem. 2017;21(4):1-7.

9. Diagnóstico de enfermagem da NANDA: definições e classificação 2015-2017. Porto Alegre: Artmed; 2015.

10. DIAS OV, et al. Diabetes mellitus em montes claros: inquérito de prevalência autorreferida. Revista Brasileira em Promoção da Saúde. 2016;29(3):406-413.

11. SOCIEDADE BRASILEIRA DE DIABETES. Diretrizes da Sociedade Brasileira de Diabetes 2017-2018. Organização José Egídio Paulo de Oliveira, Renan Magalhães Montenegro Junior, Sérgio Vencio. São Paulo: Clannad; 2017.

12. FARIA HTG, et al. Adesão ao tratamento em diabetes mellitus em unidades da Estratégia Saúde da Família. Revista da Escola de Enfermagem USP. 2014;48(2):257-63.

13. INSTITUTO BRASILEIRO DE GEOGRAFIA E ESTATÍSTICA (IBGE). Pesquisa de Orçamentos Familiares 20082009: análise da disponibilidade domiciliar de alimentos e do estado nutricional no Brasil. Rio de Janeiro: IBGE; 2011.

14. LINDEMANN IL, et al. Dificuldades para alimentação saudável entre usuários da atenção básica em saúde e fatores associados. Revista Ciência \& Saúde Coletiva. 2016;21(2):599-610.

15. MENDES EV. As redes de atenção à saúde. 2. ed. Brasília: Organização Pan-Americana da Saúde; 2011.

16. MORESCHI C, et al. Ações das equipes da ESF para a qualidade de vida das pessoas com diabetes. Ciência, Cuidado e Saúde. 2018;17(2):1-8.

17. MOTTA MDC, et al. Educação em saúde junto a idosos com Hipertensão e diabetes: estudo descritivo. Revista UNINGÁ REVIEW. 2014;18(2):48-53.

18. NETA DSR, et al. Adesão das pessoas com diabetes mellitus ao autocuidado com os pés. Revista Brasileira de Enfermagem. 2015;68(1):111-6.

19. ROSA RDS, et al. Disease and Economic Burden of Hospitalizations Attributable to Diabetes Mellitus and Its Complications: A Nationwide Study in Brazil. Int. J. Environ. Res. Public Health. 2018;15(1):294-299.

20. SEOANE AF, FORTES PAC. Percepção de médicos e enfermeiros de unidades de assistência médica ambulatorial sobre humanização nos serviços de saúde. Revista Saúde Sociedade; 2014.

21. SMELTZER S, BARE BG. Brunner\&Suddarth: tratado de enfermagem médico-cirúrgica. Rio de Janeiro: Guanabara Koogan; 2002.

22. WORLD HEALTH ORGANIZATION. Global report on diabetes. Disponível em: <http://www.who.int>. Acesso em: 20 abr. 2018. 